\title{
Concomitant Coracoid Process Fracture with Bony Bankart Lesion Treated with the Latarjet Procedure
}

\author{
Seung Gi Min ${ }^{1}$, Dong Hyun Kim ${ }^{1}$, Ho Seok Lee ${ }^{2}$, Hyun Joo Lee ${ }^{1}$ Kyeong Hyeon Park ${ }^{1}$, Jong Pil Yoon ${ }^{1}$ \\ ${ }^{1}$ Department of Orthopaedic Surgery, School of Medicine, Kyungpook National University, Daegu, Korea \\ ${ }^{2}$ Department of Radiology, School of Medicine, Kyungpook National University, Daegu, Korea
}

\begin{abstract}
Bony lesions of the glenoid and Hill-Sachs lesions are the most common injuries after a first-time traumatic shoulder dislocation. However, fracture of the coracoid process after traumatic shoulder dislocation is rare. A single, open surgical procedure could be performed by a Latarjet procedure using a fractured fragment of the coracoid process. If a fracture of the coracoid process is associated with a traumatic anterior shoulder dislocation, the Latarjet procedure may be the most appropriate surgical option.
\end{abstract}

Keywords: Shoulder dislocation; Bankart lesion; Coracoid process

A Bankart lesion is an injury of the anterior-inferior glenoid labrum of the shoulder due to anterior shoulder dislocation and bony Bankart is a Bankart lesion that includes a fracture of the anterior-inferior glenoid cavity of the scapula bone. A surgeon can consider operative treatment using either internal fixation or the Latarjet procedure when the acute glenoid rim defect is more than $5 \%$ in an active patient [1]. We present an appropriate surgical option that is suitable for patients with concomitant coracoid process fracture with bony Bankart lesions in acute traumatic shoulder dislocation.

\section{CASE REPORT}

This study was approved by the Institutional Review Board of Kyungpook National University Hospital (IRB No. KUNH 201907-001).
A 60-year-old man who was employed as a daily construction worker presented to the emergency department with trauma to his left shoulder and arm. He had fallen from a 3-m height while working and complained of pain in his left shoulder, wrist, and elbow. On physical examination, there was tenderness over the anterior aspect of the shoulder associated with pain-induced limitation in the range of motion. Plain radiography showed fractures of the distal radius and olecranon. An axillary lateral view of the left shoulder showed anterior subluxation of the humeral head and a Hill-Sachs lesion with a coracoid process fracture (Fig. 1). A computed tomography scan revealed a glenoid bone defect in addition to the above mentioned image findings (Fig. 2). An additional magnetic resonance imaging scan displayed not only a Hill-Sachs lesion of the humeral head and detachment of the anterior labrum with osseous involvement, but also a massive tear of the supraspinatus and infraspinatus tendons (Fig. 3). 

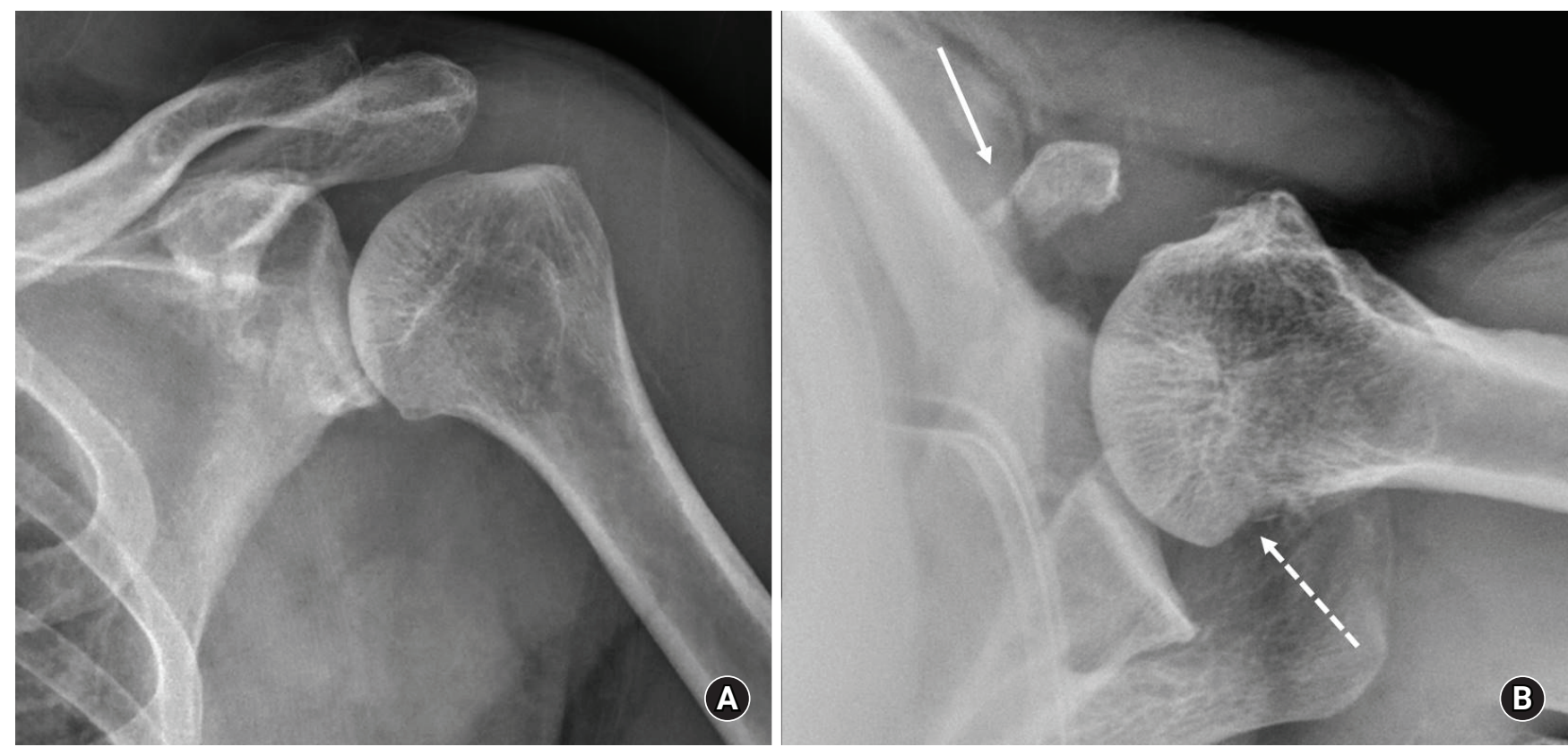

Fig. 1. Shoulder radiographs. (A) Anterior-posterior view. Humeral head inferior subluxation and abnormal coracoid process contour. (B) Axillary lateral view. Coracoid process fracture line (white arrow), Hill-Sachs lesion (white dotted arrow) and humeral head anterior subluxation.
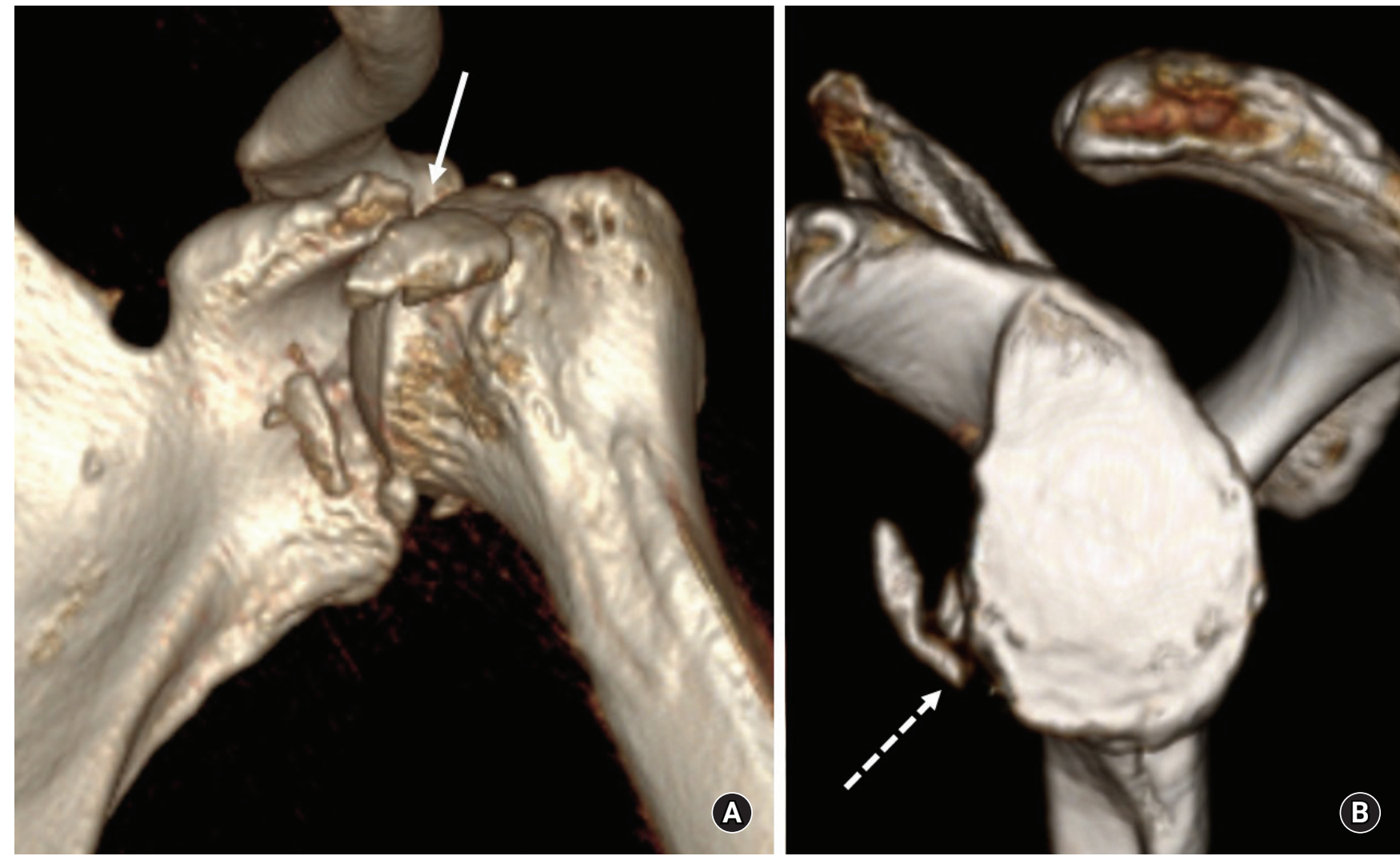

Fig. 2. Three-dimensional computed tomography. (A) Coracoid process tip avulsion fracture (white arrow) and humeral head anterior subluxation. (B) Anterior-inferior glenoid rim fracture (white dotted arrow). 

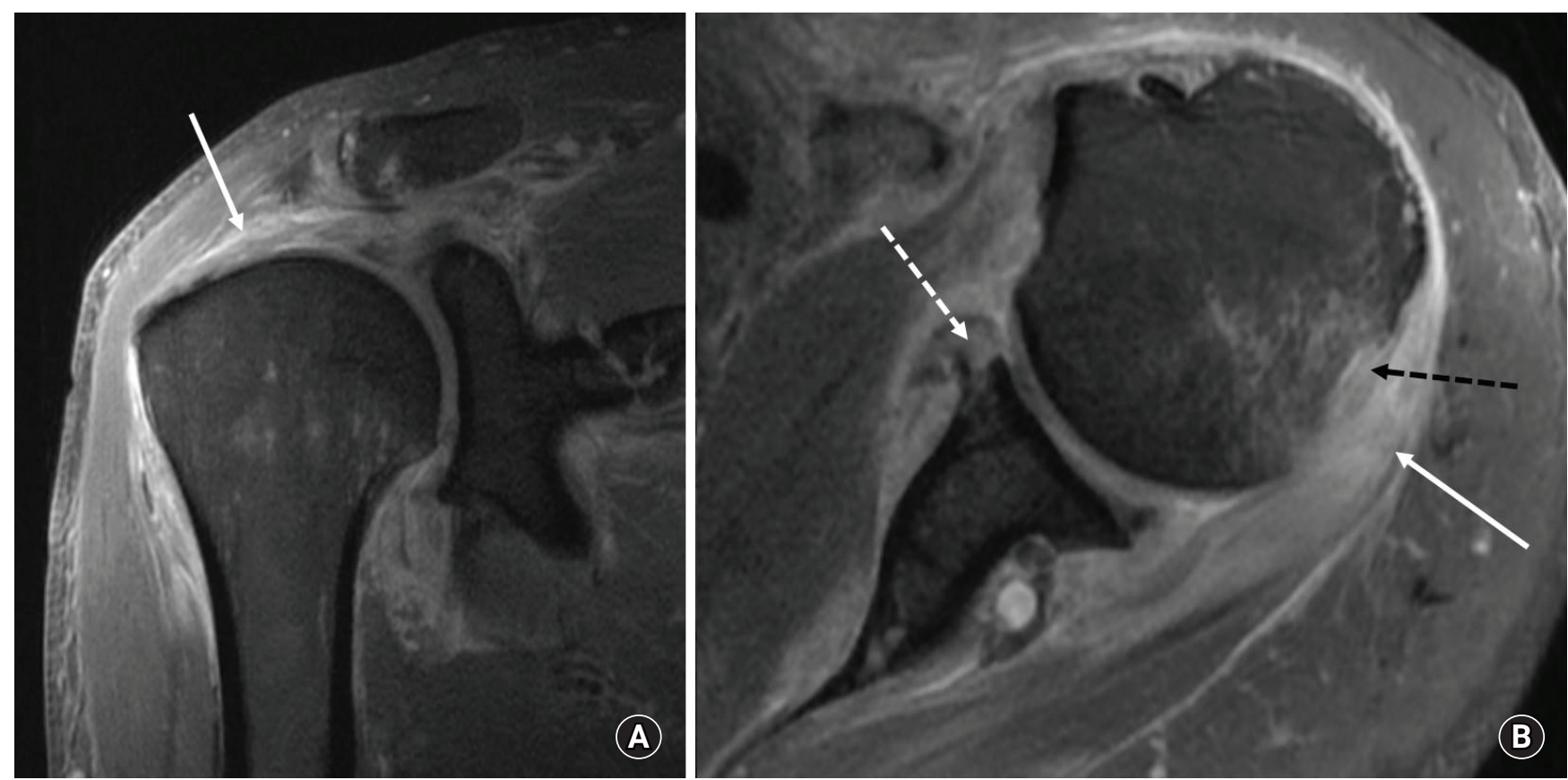

Fig. 3. Shoulder magnetic resonance imaging images. (A) Coronal view. (B) Axial view. Massive tear of supraspinatus and infraspinatus tendons (white arrows), bony Bankart lesion (white dotted arrow), Hill-Sachs lesion (black dotted arrow).
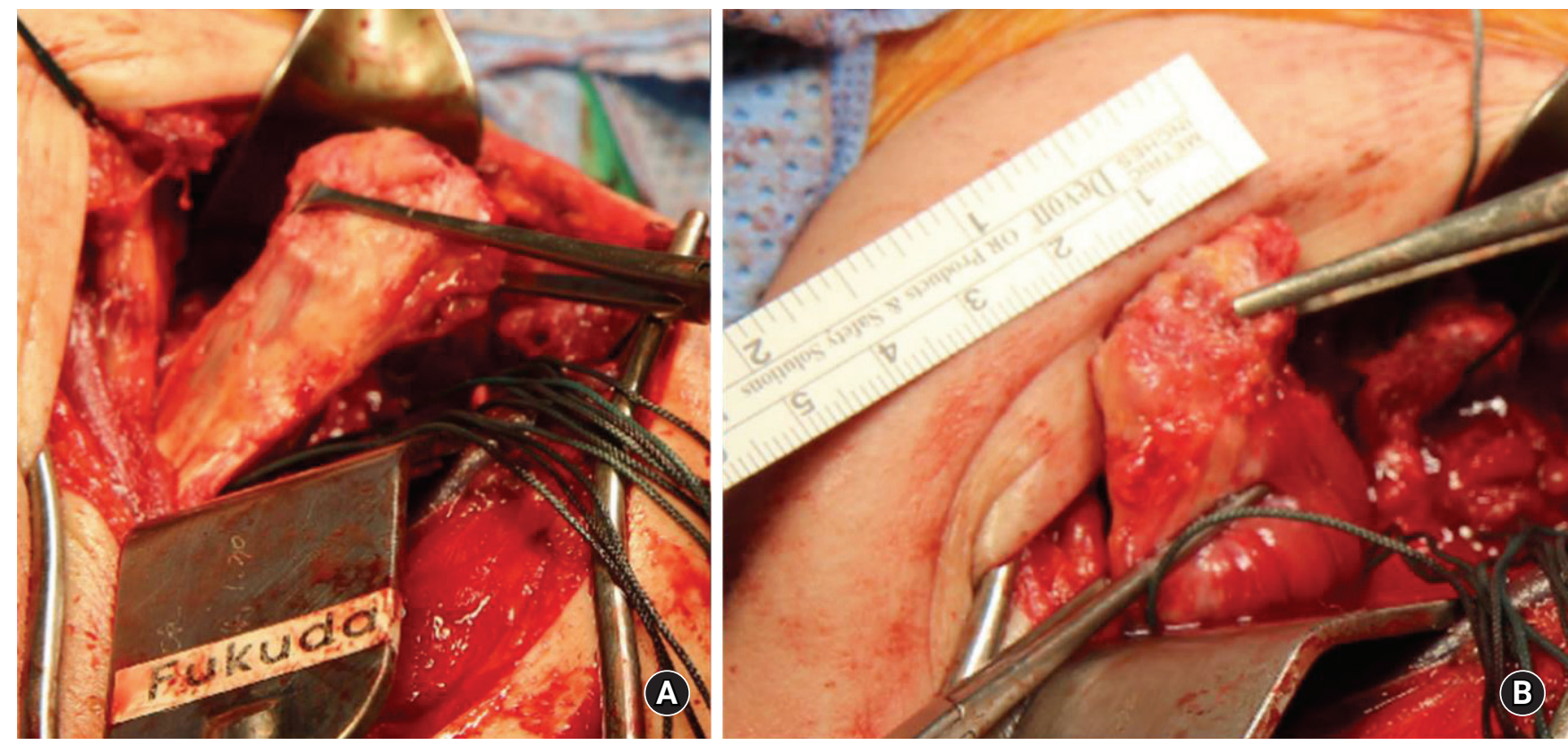

Fig. 4. (A) Identified coracoid fracture fragment and intact conjoined tendon. (B) An about 2.5-cm-sized coracoid fracture fragment.

Two days after the injury, open reduction and internal fixation were performed for olecranon and distal radius fractures. We decided to perform an open Latarjet procedure using the fractured coracoid process fragment. The operation was performed in the beach chair position. The surgical site was exposed through the deltopectoral approach. After preparation and sectioning of the clavipectoral fascia, we identified the fractured coracoid fragment (Fig. 4A). The conjoined tendons were intact and attached to the coracoid fragment. The length of the coracoid fragment was about $2.5 \mathrm{~cm}$ (Fig. 4B). The coracoid fragment with attached conjoined tendons was transferred to the glenoid rim following an open Latarjet procedure (Fig. 5). We used two 4.5-mm-diameter head- 


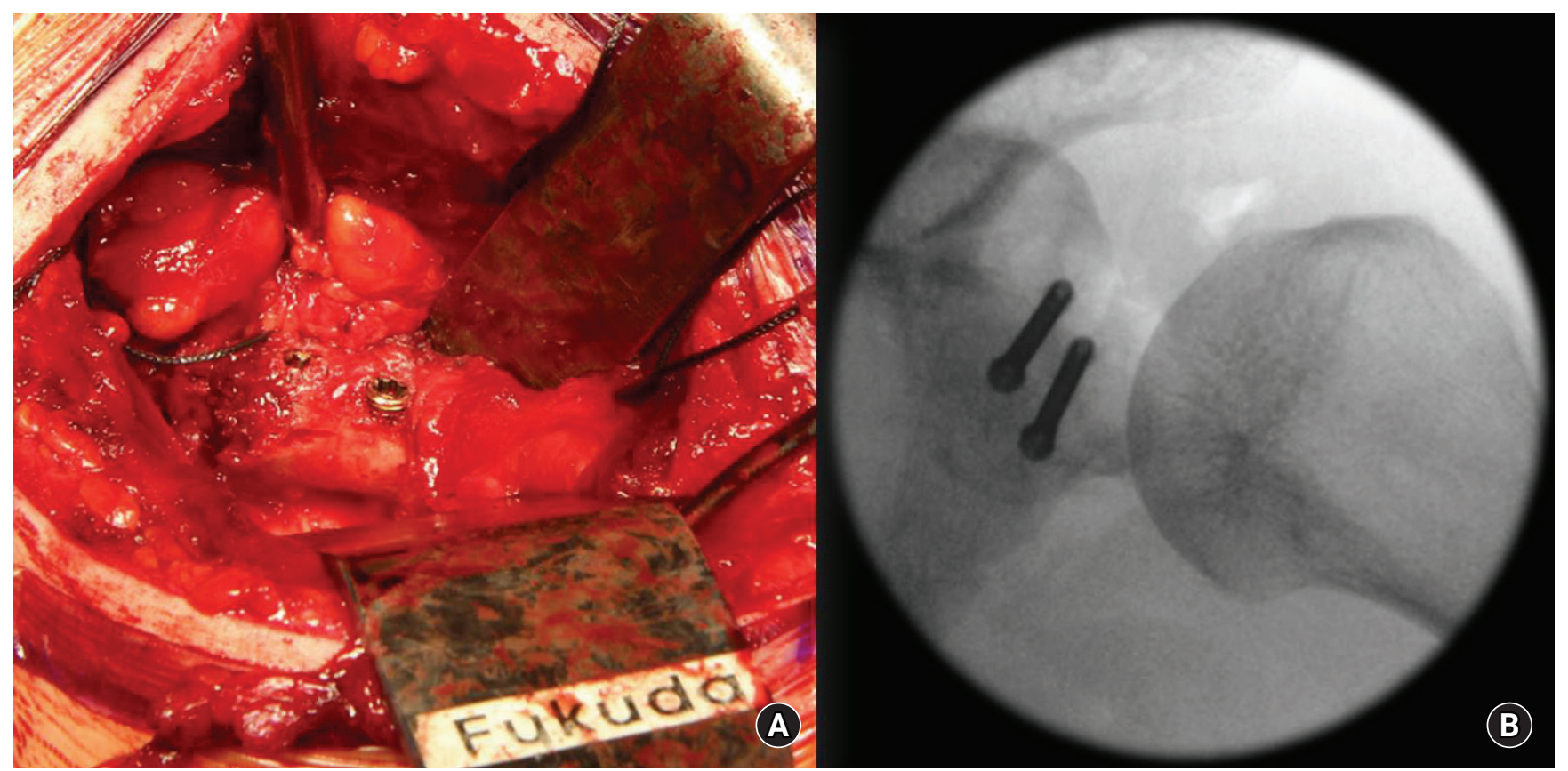

Fig. 5. (A) Transferred coracoid fragment and fixed to glenoid rim using two screws. (B) Fluoroscopic image.
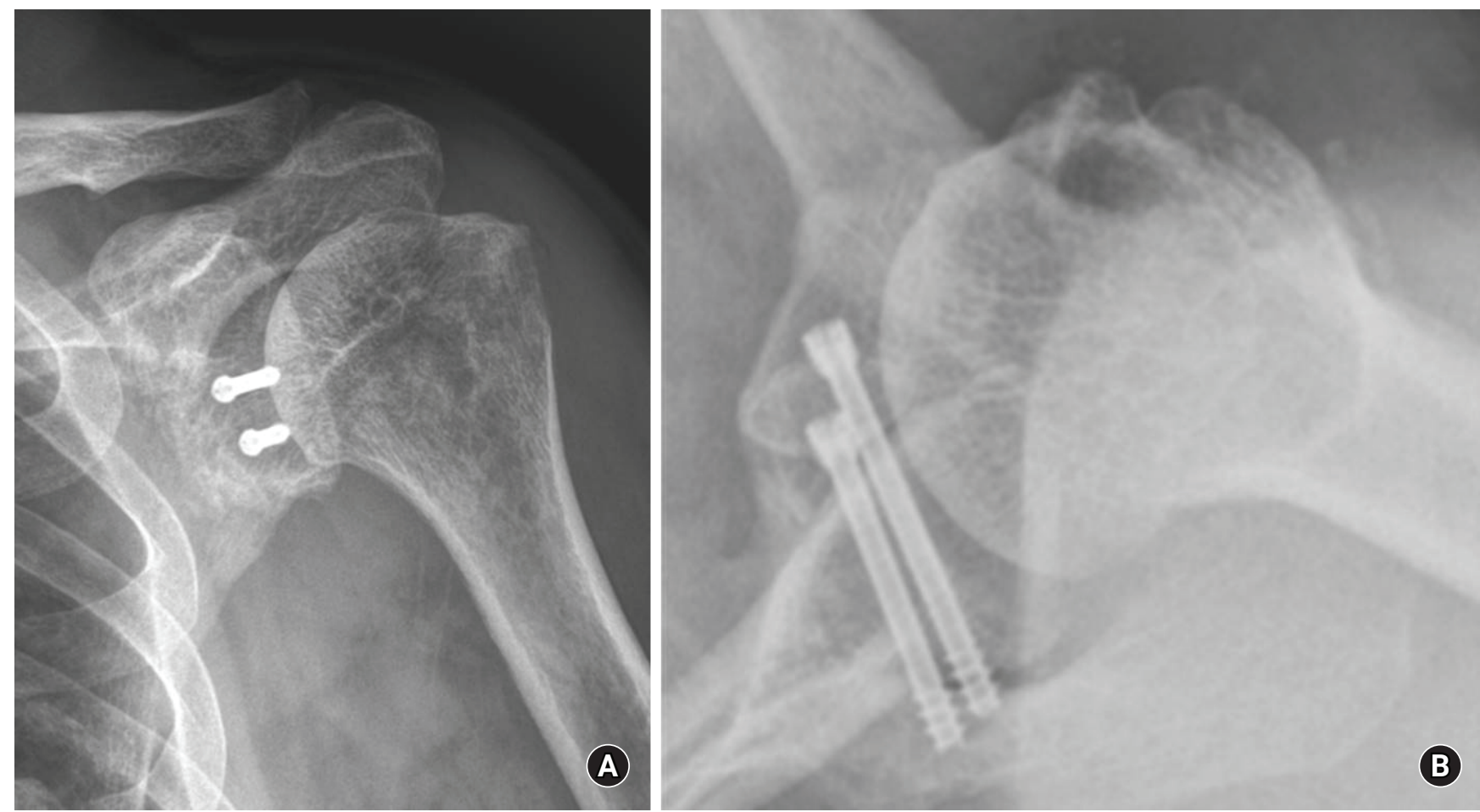

Fig. 6. Postoperative radiographs. (A) Anterior-posterior view. (B) Axillary lateral view.

less screws (length, $36 \mathrm{~mm}$ and $42 \mathrm{~mm}$ ) for fixation of the coracoid fragment (Fig. 6). As the torn supraspinatus and infraspinatus tendons had relatively good status and tissue quality, we repaired the rotator cuff using a suture bridge technique without excessive tension.

Two years after surgery, the patient obtained improvement of shoulder function and range of motion. He had no limitation of daily activities. Recurrent anterior dislocation did not occur during 
the follow-up period. At the final follow-up visit, the clinical outcomes were assessed using visual analog scale, 2/10; Simple Shoulder Test, 10/12; University of California at Los Angeles Shoulder Score, 31/35; and Constant score, 86/100.

\section{DISCUSSION}

Bony lesions of the glenoid and Hill-Sachs lesions are the most common injuries after a first-time traumatic shoulder dislocation [2-6]. However, fracture of the coracoid process after traumatic shoulder dislocation is rare $(0.8 \%-2 \%)[7,8]$. Generally, an acute glenoid rim defect caused by first-time shoulder dislocation can be treated operatively using a bone graft when the glenoid defect is larger than 25\% [9]. A surgeon can consider operative treatment using either internal fixation or the Latarjet procedure when the acute glenoid rim defect is larger than $5 \%$ in an active patient [1]. A type II coracoid process fracture can be treated conservatively, but some studies report that a coracoid pseudoarthrosis can occur when there is a concomitant anterior shoulder dislocation [10]. A few cases of shoulder dislocations with a simultaneous fracture of the coracoid process have been previously reported in the literature [11]. Ogawa et al. $[12,13]$ classified fractures of the coracoid process into two types based on the coracoclavicular ligaments. They recommended surgical treatment in type I fractures (fractures proximal to the coracoclavicular ligaments). Type II fractures (fractures distal to the coracoclavicular ligaments) can be treated conservatively. However, Kälicke et al. [10] reported that a coracoid pseudoarthrosis could be caused by tension of the conjoined tendons in an anterior shoulder dislocation with a concomitant type II coracoid fracture. Therefore, surgical treatment should be considered to prevent pseudoarthrosis or nonunion in type II fractures with displacement greater than $5 \mathrm{~mm}$.

The mechanism of injury in our case probably resulted from direct trauma of the dislocated humeral head against the glenoid rim and coracoid process. The fracture of the coracoid process was type II, and the fractured fragment measured about $2.5 \mathrm{~cm}$. The glenoid bone defect was $15 \%$ by Sugaya's method [14], and anterior subluxation of the humeral head was sustained in more than $50 \%$ of the glenoid [15,16]. A defect involving more than $20 \%$ to $25 \%$ of the glenoid bone has historically been considered critical bone loss causing a recurrent anterior shoulder dislocation. Therefore, a Latarjet procedure or bone graft from the iliac bone is a surgical option for a severe glenoid bone defect. The treatment choice in cases involving borderline defects of the glenoid (15\%-20\%) is controversial and left to the surgeon's preference [17]. In the case of an isolated coracoid process fracture treated surgically, screw fixation, plate fixation, or tension band wiring are considered as treat- ment methods [12,18].

To treat glenoid bone loss in a first-time shoulder dislocation concomitant with a coracoid process fracture and a rotator cuff tear, like the present case, by one-time surgery, the Latarjet procedure can be an ideal option for solving all the problems in one step. The Latarjet procedure has a sling effect caused by the conjoined tendons as well as bony augmentation of the glenoid rim using the coracoid process, and it is used for cases of failed anterior shoulder instability or a large bony defect of the glenoid. But, in the case of an elderly patient with poor rotator cuff function, surgeons must carefully consider the treatment options, because the Latarjet procedure may compromise further surgeries, like reverse total shoulder arthroplasty for irreparable massive rotator cuff tear. The previous literature reported only one case where the Latarjet procedure was performed in a patient with chronic recurrent anterior shoulder instability with a fracture of the coracoid process [19]. The present report described the first case of Latarjet procedure in an acute concomitant bony Bankart lesion with a coracoid process fracture.

We reported a surgical method that can solve concomitant lesions by a one-time operation. If a fracture of the coracoid process is associated with a traumatic anterior shoulder dislocation, the Latarjet procedure may be the most appropriate surgical option.

\section{ORCID}

Seung Gi Min

Dong Hyun Kim

Ho Seok Lee

Hyun Joo Lee

Kyeong Hyeon Park

Jong Pil Yoon

https://orcid.org/0000-0003-4343-1022 https://orcid.org/0000-0001-9078-5953 https://orcid.org/0000-0003-3670-0693 https://orcid.org/0000-0003-2837-3434 https://orcid.org/0000-0001-7215-6176 https://orcid.org/0000-0001-6446-6254

\section{REFERENCES}

1. Spiegl UJ, Ryf C, Hepp P, Rillmann P. Evaluation of a treatment algorithm for acute traumatic osseous Bankart lesions resulting from first time dislocation of the shoulder with a two year follow-up. BMC Musculoskelet Disord 2013;14:305.

2. Antonio GE, Griffith JF, Yu AB, Yung PS, Chan KM, Ahuja AT. First-time shoulder dislocation: high prevalence of labral injury and age-related differences revealed by MR arthrography. J Magn Reson Imaging 2007;26:983-91.

3. Fujii Y, Yoneda M, Wakitani S, Hayashida K. Histologic analysis of bony Bankart lesions in recurrent anterior instability of the shoulder. J Shoulder Elbow Surg 2006;15:218-23.

4. Habermeyer P, Gleyze P, Rickert M. Evolution of lesions of the 
labrum-ligament complex in posttraumatic anterior shoulder instability: a prospective study. J Shoulder Elbow Surg 1999;8:6674.

5. Tauber M, Resch H, Forstner R, Raffl M, Schauer J. Reasons for failure after surgical repair of anterior shoulder instability. J Shoulder Elbow Surg 2004;13:279-85.

6. Yiannakopoulos CK, Mataragas E, Antonogiannakis E. A comparison of the spectrum of intra-articular lesions in acute and chronic anterior shoulder instability. Arthroscopy 2007;23:98590.

7. Hovelius L, Eriksson K, Fredin H, et al. Recurrences after initial dislocation of the shoulder: results of a prospective study of treatment. J Bone Joint Surg Am 1983;65:343-9.

8. McLaughlin HL, MacLellan DI. Recurrent anterior dislocation of the shoulder. II. A comparative study. J Trauma 1967;7:191201.

9. Kwong CA, Gusnowski EM, Tam KK, Lo IK. Assessment of bone loss in anterior shoulder instability. Ann Jt 2017;2:63.

10. Kälicke T, Andereya S, Gekle J, Müller EJ, Muhr G. Coracoid pseudarthrosis caused by anterior shoulder dislocation with concomitant coracoid fracture. Unfallchirurg 2002;105:843-4.

11. Plachel F, Schanda JE, Ortmaier R, Auffarth A, Resch H, Bogner R. The "triple dislocation fracture": anterior shoulder dislocation with concomitant fracture of the glenoid rim, greater tuberosity and coracoid process-a series of six cases. J Shoulder Elbow Surg 2017;26:e278-85.
12. Ogawa K, Matsumura N, Ikegami H. Coracoid fractures: therapeutic strategy and surgical outcomes. J Trauma Acute Care Surg 2012;72:E20-6.

13. Ogawa K, Yoshida A, Takahashi M, Ui M. Fractures of the coracoid process. J Bone Joint Surg Br 1997;79:17-9.

14. Sugaya H, Moriishi J, Dohi M, Kon Y, Tsuchiya A. Glenoid rim morphology in recurrent anterior glenohumeral instability. J Bone Joint Surg Am 2003;85:878-84.

15. Giles JW, Boons HW, Elkinson I, et al. Does the dynamic sling effect of the Latarjet procedure improve shoulder stability? A biomechanical evaluation. J Shoulder Elbow Surg 2013;22:821-7.

16. Yamamoto N, Muraki T, An KN, et al. The stabilizing mechanism of the Latarjet procedure: a cadaveric study. J Bone Joint Surg Am 2013;95:1390-7.

17. Provencher MT, Bhatia S, Ghodadra NS, et al. Recurrent shoulder instability: current concepts for evaluation and management of glenoid bone loss. J Bone Joint Surg Am 2010;92 Suppl 2:13351.

18. Anavian J, Wijdicks CA, Schroder LK, Vang S, Cole PA. Surgery for scapula process fractures: good outcome in 26 patients. Acta Orthop 2009;80:344-50.

19. Schneider MM, Balke M, Koenen P, Bouillon B, Banerjee M. Avulsion fracture of the coracoid process in a patient with chronic anterior shoulder instability treated with the Latarjet procedure: a case report. J Med Case Rep 2014;8:394. 